\title{
RETRACTED ARTICLE: The decline of glomerular function is not always associated with the development of micro- and macroalbuminuria in hypertensive patients with type 2 diabetes
}

\author{
R. Nosadini • A. Carboni • A. Manconi - F. Angius • \\ S. Caria $\cdot$ S. Cherchi $\cdot$ A. Satta $\cdot$ R. Faedda $\cdot$ D. Obinu $\cdot$ \\ M. Nieddu • A. Carraro • G. C. Tonolo
}

Received: 7 January 2008 / Accepted: 21 May 2008

(C) The Author(s) 2008

This article has been withdrawn due to copyright infringement via plagiarism.

This article has been withdrawn due to copyright infringement via plagiarism.

R. Nosadini $(\bowtie)$

Department of Endocrinology and Metabolic Diseases,

Internal Medicine Department, University of Sassari,

Viale San Pietro 8,

07100 Sassari, Italy

e-mail: noscia@tin.it

A. Carboni $\cdot$ A. Manconi $\cdot$ F. Angius $\cdot$ S. Caria $\cdot$ S. Cherchi $\cdot$

D. Obinu $\cdot$ M. Nieddu $\cdot$ G. C. Tonolo $(\triangle)$

General Hospital ASL 2, Via Canova,

Olbia, Italy

e-mail: noscia@tin.it

\footnotetext{
A. Carraro

General Hospital Mirano,

Venice, Italy
}

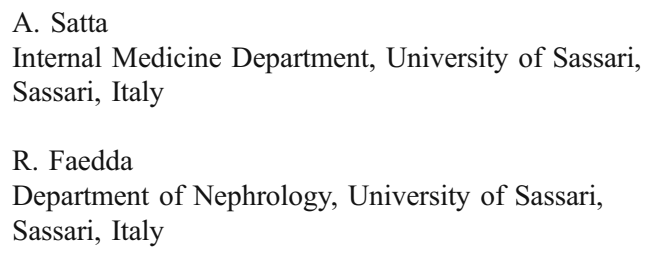

